\title{
Blast Responses and Vibration of Flood-Defense Structures under High-Intensity Blast Loadings
}

\author{
Yonghee Ryu, ${ }^{1}$ WooYoung Jung $\left(\mathbb{D},{ }^{2}\right.$ HoYoung Son, ${ }^{3}$ and Bu-Seog Ju $\mathbb{D}^{3}$ \\ ${ }^{1}$ Samsung Heavy Industries, Pangyo, Bundang-gu, Sungnam-si, Gyungki-do 13486, Republic of Korea \\ ${ }^{2}$ Department of Civil Engineering, Gangneung-Wonju National University, Gangneung 25457, Republic of Korea \\ ${ }^{3}$ Department of Civil Engineering, Kyung Hee University, Yongin-si, Gyeonggi-do 17104, Republic of Korea \\ Correspondence should be addressed to Bu-Seog Ju; bju2@khu.ac.kr
}

Received 25 July 2017; Accepted 31 January 2018; Published 27 February 2018

Academic Editor: Giuseppe Brandonisio

Copyright (C) 2018 Yonghee Ryu et al. This is an open access article distributed under the Creative Commons Attribution License, which permits unrestricted use, distribution, and reproduction in any medium, provided the original work is properly cited.

\begin{abstract}
This study presented the blast behavior of flood-defense structures subjected to high-intensity loadings such as blast shock waves. In order to understand the blast behavior of weir structures, PHAST program was used to predict blast loadings in consideration of material reactivity and congestion levels. Environment factors such as weather data and atmospheric parameters were also considered in this study. Then, nonlinear dynamic analyses were performed using the ABAQUS platform to evaluate structural responses and blast vibration of concrete weir structures subjected to various types of blast loadings, due to uncertainties of the magnitude and durations of blast loads as a function of distance from the explosion. It was shown that the blast damage to concrete weir structure was significantly influenced by congestion levels or material reactivity. Also, the stress concentration under blast loading was observed at the connection area between the concrete weir body and stilling basin.
\end{abstract}

\section{Introduction}

Critical infrastructures such as bridges, dams, nuclear power plants, lifelines, and buildings can be exposed to terrorist attacks and chemical explosions. The Pentagon and World Trade Center in 2001, the Australian Embassy in Jakarta, Indonesia in 2004, and Bangkok City, Thailand in 2015, are the sites of infamous terrorist attacks. Representing a much higher likelihood of explosion than terrorist conspiracy are chemical and petrochemical plants, due to explosive materials and hot work which is frequent ignition sources. In China, for example, there have been two recent major explosions at petrochemical plants: in Jilin in 2005, which killed six people and necessitated the evacuation of thousands more, and in Guangxi in 2008, which killed 16 people (https://en.wikipedia.org/). Moreover, and more recently still, the devastation due to the strongest recent explosion at Tianjin in China occurred at a warehouse containing hazardous materials such as calcium carbide, sodium cyanide, and sodium nitrate, killing more than 100 people and hospitalizing about 100 others.
Many researchers [1-3] have recognized the need to address, in the design stage, the problem of the protection for critical structures from high-intensity dynamic events generated by strong explosions. Nog et al. [4] studied blast-loading effects on structures and the blast-wave mechanism in free air. Dharmasena et al. [5] evaluated the dynamic and mechanical responses of metallic honeycomb sandwich panel structures under high-intensity loading using ABAQUS/Explicit based on experimental tests. Also, reliability analyses of reinforced concrete subjected to blast loadings have been conducted, by Low and Hao [6]. In addition, Xiangdi et al. [7] introduced a new, computational fluid dynamics- (CFD-) based method of risk determination for gas-explosion hazards in China. Furthermore, in recent years, infrastructure systems were designed to withstand extreme loads such as earthquake or blast. Fujikura et al. [8] described the behavior of bridge pier systems under multihazard scenarios such as seismic and blast loadings based on FEMA [9] and FHWA [10]. The inelastic deformation of bridge pier was experimentally and numerically investigated by using a simplified single degree of freedom system. Li et al. [11] evaluated the performance 


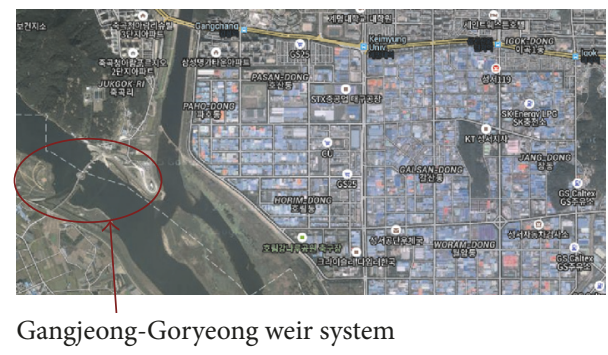

FIGURE 1: Gangjeong-Goryeong weir system near Daegu Seongseo Industrial Complex in Korea.

of overhead protection structures subjected to blast loads following the procedures in ASCE guidelines in which finite element analysis (FEA) was conducted by the ABAQUS platform. In Korea, on the other hand, performance evaluation of flood-defense dam/weir structures subjected to blast loads has seldom been carried out. The focus of the present study, therefore, was the characterization of the performance of weir-infrastructure systems under various types of blast loads. More specifically, blast-loading explosive weight and stand-off distance were considered as intensity uncertainties in evaluating soil-structure interaction in accordance with material uncertainties in Finite Element (FE) models. Additionally, in order to generate a scenario involving blast loads related to man-made hazards, PHAST [12] as a consequencemodeling program was employed for several important factors such as humidity, temperature, material reactivity, and congestion. This validated program [13-15] is widely used to calculate the blast loadings using a Baker-Strehlow-Tang (BST) model in which the blast model is also validated by experimental data $[16,17]$. Three types of quantitative explosion analyses, low, medium, and high material reactivity and congestion levels, were performed, and PHAST-based blast time histories corresponding to those levels were derived. Finally, the sensitivity studies were conducted to address the relative effects of material uncertainties on the weir structure as modeled in ABAQUS [18].

\section{Selected Target Areas and Flood-Defense Structures}

Extreme loads can cause failure of flood-defense structures such as dams and weir systems. The 2011 Japan earthquake and massive tsunami, for example, the most destructive natural catastrophe in that country's history, caused extensive damage to an entire regional area even though there had been a seawall in place for protection of infrastructure. In recent years in Korea, several concrete weir structures for flood (or drought) control have been constructed, some of which are located nearby major industrial complexes including petrochemical plants and Liquefied Petroleum Gas (LPG) stations. To assess damage to weir structures and improve the performance of flood-defense structures under blast loads, the Gangjeong-Goryeong weir system located near the Daegu Seongseo Industrial Complex in Korea was selected for evaluation in this study (see Figure 1). The Gangjeong-Goryeong hydraulic system's designed pool elevation is $19.50 \mathrm{~m}$ for the

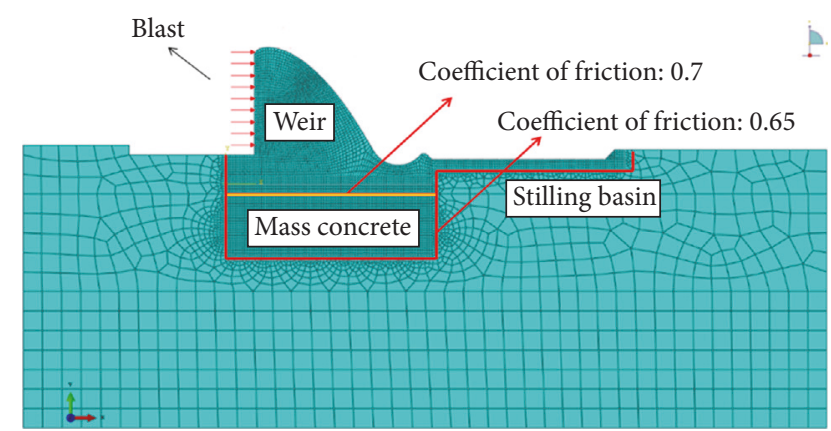

Figure 2: The figure shows FE model of Gangjeong-Goryeong weir hydraulic system reproduced from Ju and Jung $[19,20]$, [under the Creative Commons Attribution License/Public Domain].

nonoverflow section and $9.47 \mathrm{~m}$ for the overflow section, and the storage capacity for generation of electric power and flood/drought control is 92.3 million $\mathrm{m}^{3}$. The material properties of the weir structures and soil foundations are as follows: elastic modulus of weir body and mass concrete, 26,637 MPa and 24,579 MPa, respectively; elastic modulus of steel, 200,000 MPa; unit weight of weir and mass concrete, $2350 \mathrm{~kg} / \mathrm{m}^{3}$; design strength of weir body and mass concrete, $24 \mathrm{MPa}$ and $18 \mathrm{MPa}$; Poisson's ratio for both weir and mass concrete, 0.167. Further details on some of those material properties are provided in Table $1[19,20]$.

\section{Numerical Model of Weir Structures}

In order to understand the behavior of weir structure as a flood-defense structure subject to high-intensity loadings such as explosions, a numerical FE model was developed using the ABAQUS [18] platform in this study. The FE model of the Gangjeong-Goryeong weir hydraulic system shown in Figure 2 was completed according to the 4-node bilinear plane strain quadrilateral element (CPE4R) for the weir, mass concrete, and soil foundations. The dam/weir is a continuous structure in which the performance of the dam/weir against the blast loading can be assumed to be the plain strain behavior. As one dimension is very large compared to the other dimensions, the principal strain in the direction of the longest dimension is constrained and assumed as zero. For the steel rebar, a 2D truss element was used. The soil foundations specifically were modeled based on Mohr-Coulomb material with perfectly plastic behavior. Accordingly, there were two classifications of FE model: (1) a 2D linear elastic model for the weir and (2) a 2D nonlinear model for soil-structure interaction based on the isotropic Coulomb friction material in ABAQUS. It should be noted that any FE model of a weir-structure system for blast or explosion must take into account hydrostatic and dynamic forces such as uplift pressure of piecewise liner distribution, hydrostatic pressure corresponding to the water level, and hydrodynamic pressure based on Westergaard's added-mass method [21]. The fundamental frequency of the weir structure is $1.24 \mathrm{~Hz}$. The detail discussions regarding the FE modeling of the weir-structure system can be found in Ju and Jung [19]. 
TABLE 1: Material properties of weir structures (after Ju and Jung $[19,20]$ ).

\begin{tabular}{lccc}
\hline Structures & Elastic modulus $(\mathrm{MPa})$ & Poisson's ratio & ${\text { Density }\left(\mathrm{t} / \mathrm{mm}^{3}\right)}^{\text {' }}$ \\
\hline Weir body & 26,637 & 0.167 & $2.4 E-9$ \\
Mass concrete & 24,579 & 0.167 & $2.4 E-9$ \\
Steel & 200,000 & 0.25 & $7.85 E-9$ \\
Soil layer I & 2 & 0.4 & $1.7 E-9$ \\
Soil layer II & 25 & 0.4 & $1.9 E-9$ \\
Soil layer III & 2,000 & 0.3 & $2.4 E-9$ \\
\hline
\end{tabular}

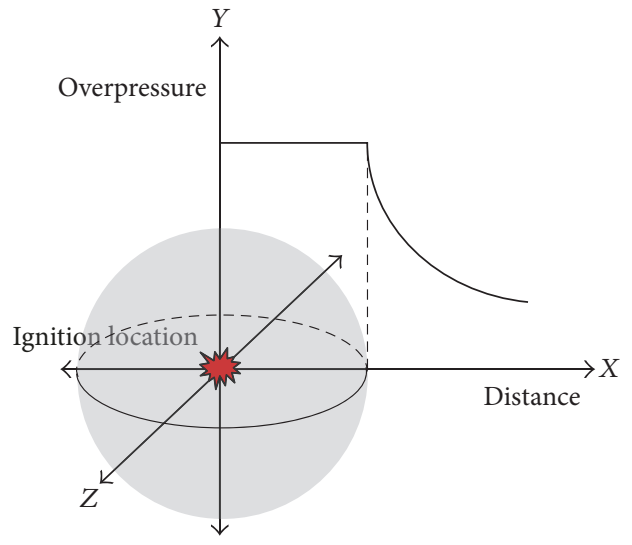

FIGURE 3: BST blast curve based on idealized vapor cloud explosion.

\section{Blast Loading from Gas Explosion}

An explosion is a sudden extreme energy release generating high temperatures and blast pressures. Deflagration leads to high pressure ( $~ 5$ bar $)$ and flame propagating at the speed of sound via heat transfer in congested areas. Detonation is a more severe explosion leading to very high pressure ( 20 bar $)$ and flame speed $(1,600 \sim 2,000 \mathrm{~m} / \mathrm{s})$, not only inside congested areas but also outside [22]. Such high pressures can result, even at long distances, in structural damage. The Buncefield disaster in December 2005 was a major accident caused by a series of explosions at storage tanks in England. Serious damage associated with blast overpressures was observed in facilities and buildings surrounding the explosion site [23]. The Texas City Refinery explosion in March 2005 was one of the worst industrial disasters in recent US history; indeed, houses as far away as $1.2 \mathrm{~km}$ from the refinery incurred damage [24].

Blast-loading prediction has been performed by researchers using experimental tests and computational fluid dynamic (CFD) simulations. Baker et al. [25] proposed an explosion model for calculation of blast pressures from vapor cloud explosions (VCE), defining potential explosion sites with respect to the explosion size and severity as well as the maximum flame speed on the basis of fuel reactivity (burning velocity), confinement (degrees of freedom), and congestion (density). A new version of this model incorporating updated blast curves was renamed the Baker-Strehlow-Tang (BST) model [26]. This model was developed based on threedimensional numerical CFD simulations of VCE (see Figure 3), with overpressure and impulse curves of positive
TABLE 2: Flame speed table for BST model (after Pierorazio et al. [16]).

\begin{tabular}{lccc}
\hline Material reactivity & Low & $\begin{array}{c}\text { Congestion } \\
\text { Medium }\end{array}$ & High \\
\hline High & 0.59 & $\mathrm{DDT}^{*}$ & $\mathrm{DDT}^{*}$ \\
Medium & 0.47 & 0.66 & 1.60 \\
Low & 0.079 & 0.47 & 0.66 \\
\hline${ }^{*}$ Deflagration-to-detonation transition. & &
\end{tabular}

and negative phases provided as functions of distance for various flame speeds covering deflagration to detonation. The BST model's table of flame speed was revised based on experimental results and updated according to a confinement of 2.5D, as shown in Table 2. A series of medium-scale VCE tests were conducted to measure the flame speed for a given fuel reactivity/confinement/congestion set. $2.5 \mathrm{D}$ values were introduced by averaging between the $2 \mathrm{D}$ and $3 \mathrm{D}$ confinement values for the same set of conditions [16].

\section{Prediction of Blast Pressure Using PHAST}

The BST model predicts explosion load and impulse with reference to the flame speed table and blast curves in terms of the fuel reactivity, confinement, and congestion in the region around the release. The explosive energy is determined by the total flammable mass under the given environmental conditions. In the current study, the PHAST [12] program (v7.1) was used as the modeling environment for the evaluation of blast. The explosion scenarios involving major accidents were assumed to occur at an onshore production platform. The catastrophic rupture for various sizes of storage tanks filled with propane (flash point $-104^{\circ} \mathrm{C}$ ) was considered to be the worst case for major accidents. The weather data and atmospheric parameters in Table 3 were adapted from a study on a procedure for evaluating the consequences of accidents [27]. The ground correction factor for each scenario in the present simulations was chosen as 2 on the recommendation of a ground-effect study [17]. Figures 4 and 5 plot the results of pressure and impulse at specified distances for the various flammable masses 5, 10, 20, 40, 60, 80100 , and 200 tons. The degree of confinement was assumed to be $2 \mathrm{D}$, solid decks on multilevel process units, and more than $80 \%$ blockage by piping structures [25]. A total of 9 scenarios for three levels, high, medium, and low, of congestion and material reactivity, respectively, were considered in this study. $2.0 \mathrm{HM}$, for example, indicates 2.0 degrees of confinement, high 

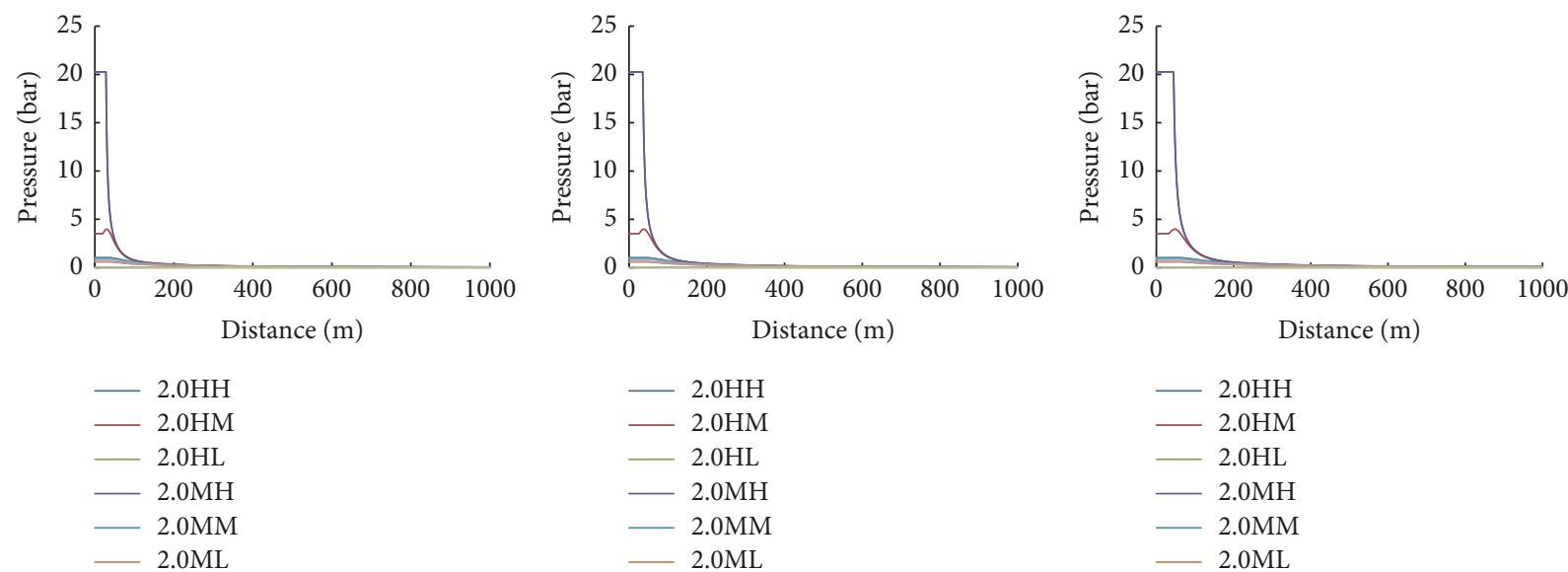

(a) 5 tons

(b) 10 tons
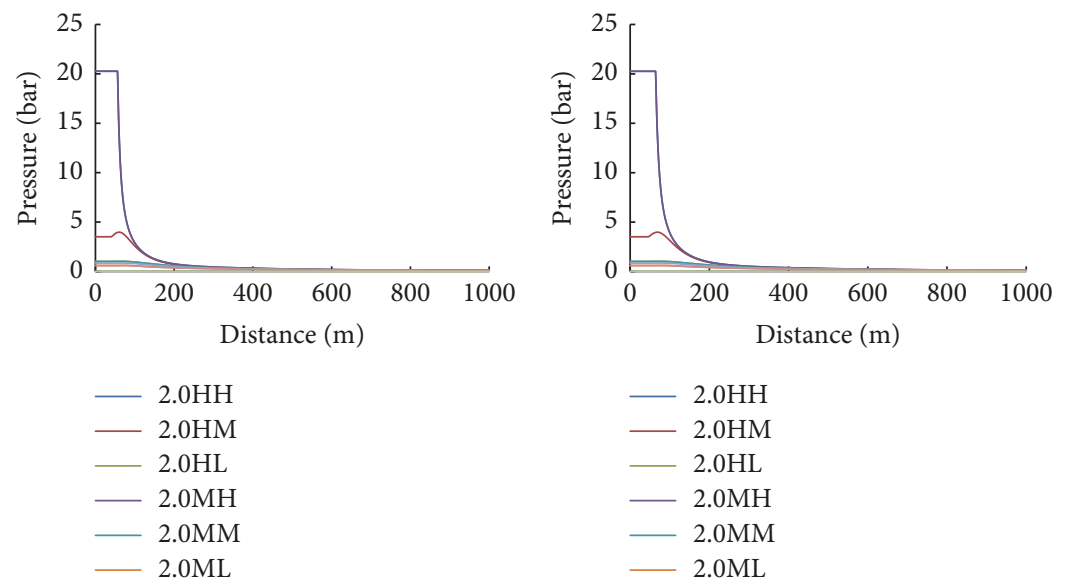

(c) 20 tons

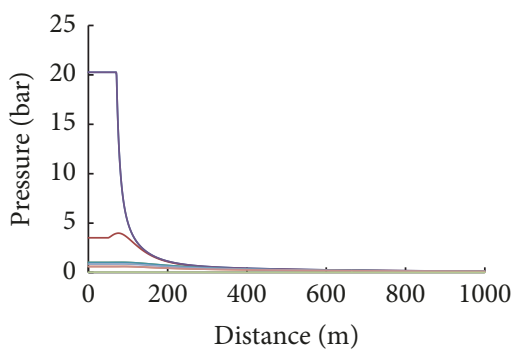

(d) 40 tons

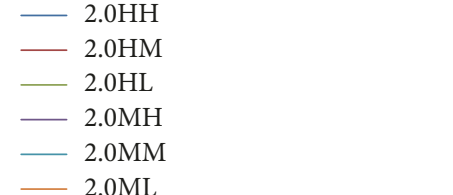

(e) 60 tons

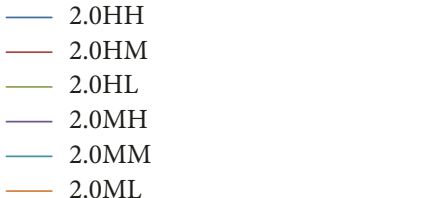

(f) 80 tons

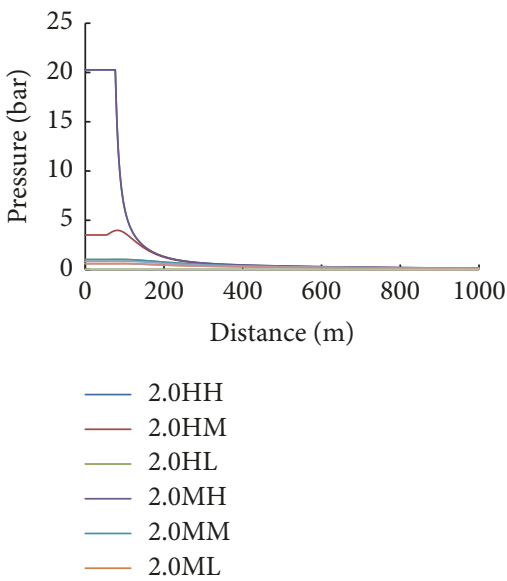

(g) 100 tons

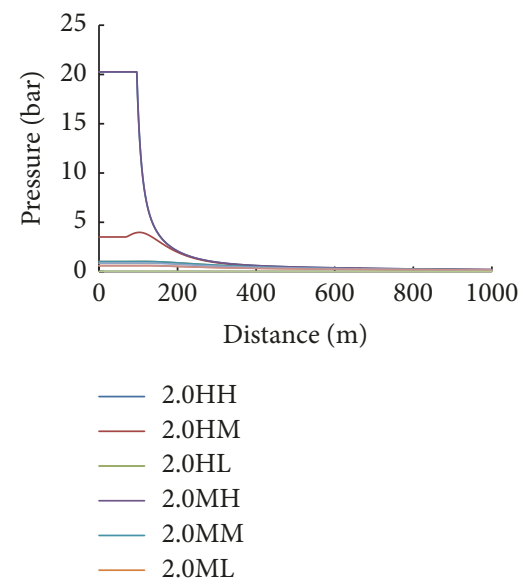

(h) 200 tons

Figure 4: Pressure versus distance for various flammable masses.

level of congestion, and medium level of material reactivity. Figure 6 represents the blast pressure results based on the BST method. The impulse is the integral of the pressure over time interval. Therefore, the blast pressure duration was simply calculated as two times the right-angled-triangle area divided by the maximum pressure at a certain distance.

\section{Blast Response and Vibration of Flood-Defense Structures under High-Intensity Blasts}

An explosion is a rapid increase in volume and release of energy in very short duration. Explosive force is released in 


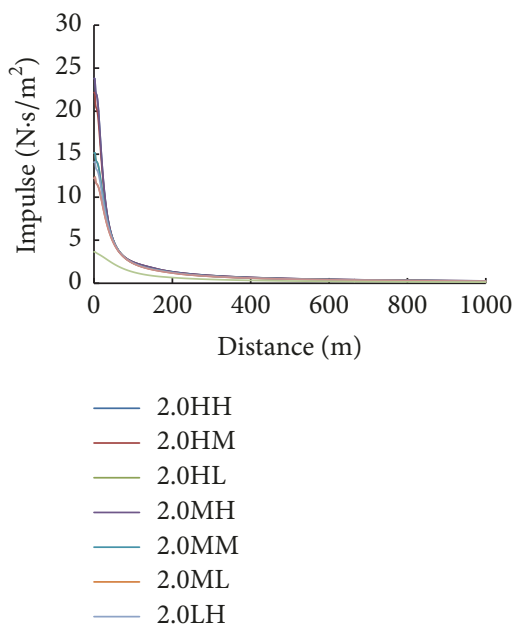

(a) 5 tons

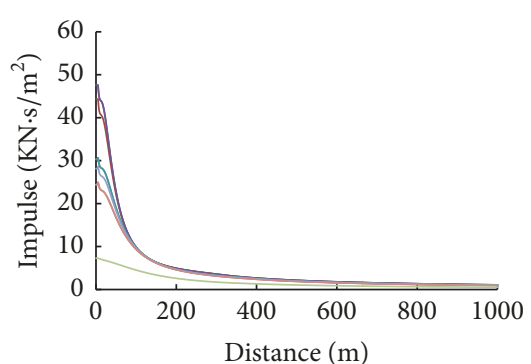

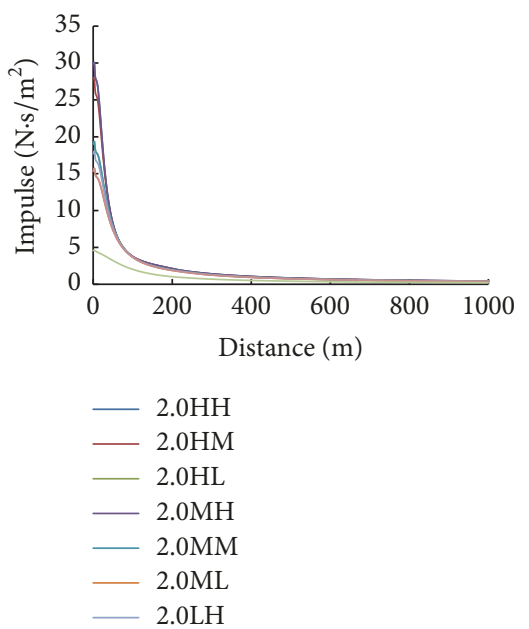

(b) 10 tons
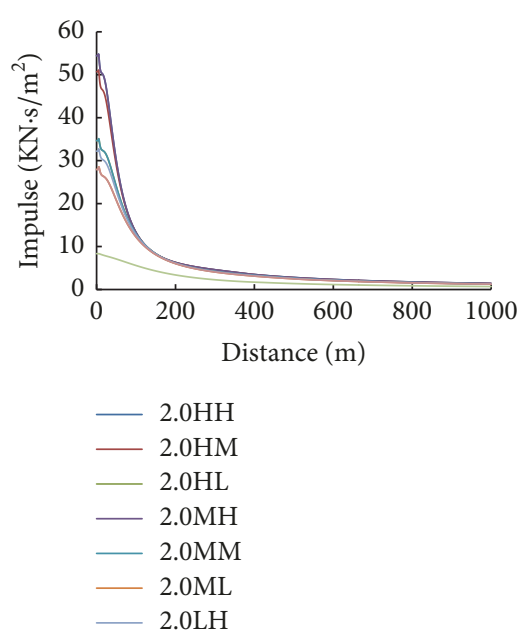

(e) 60 tons

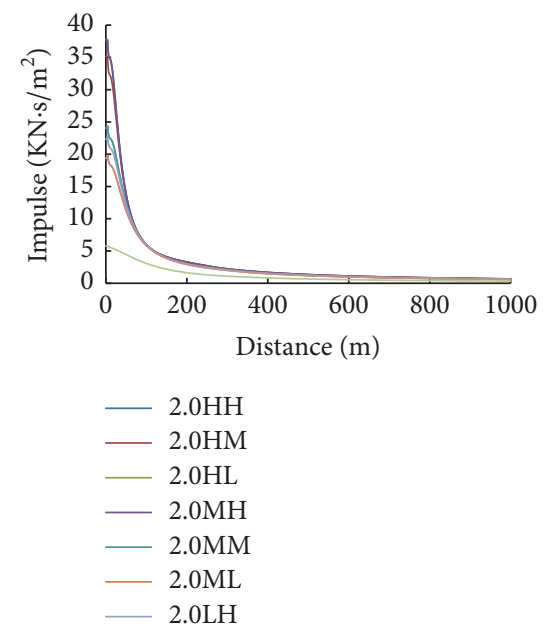

(c) 20 tons

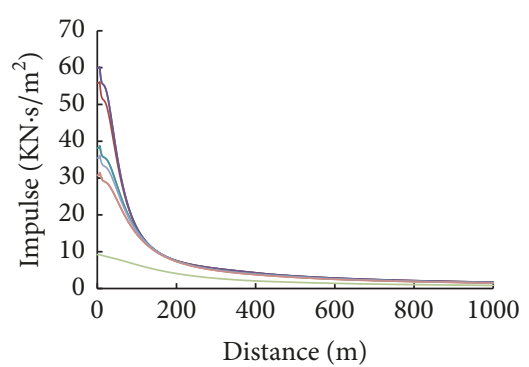

(d) 40 tons

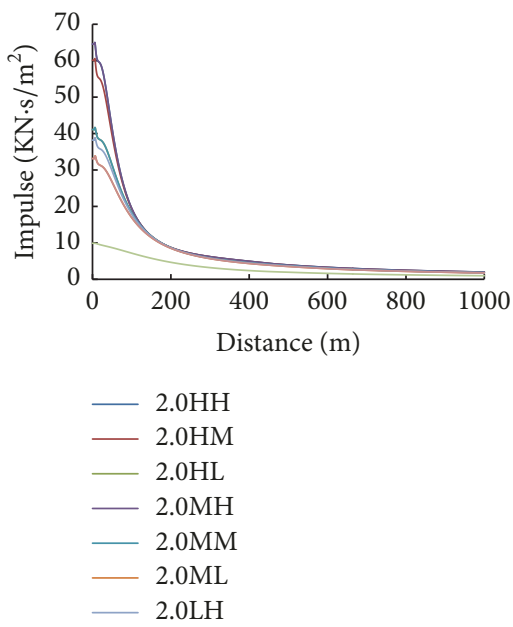

(g) 100 tons
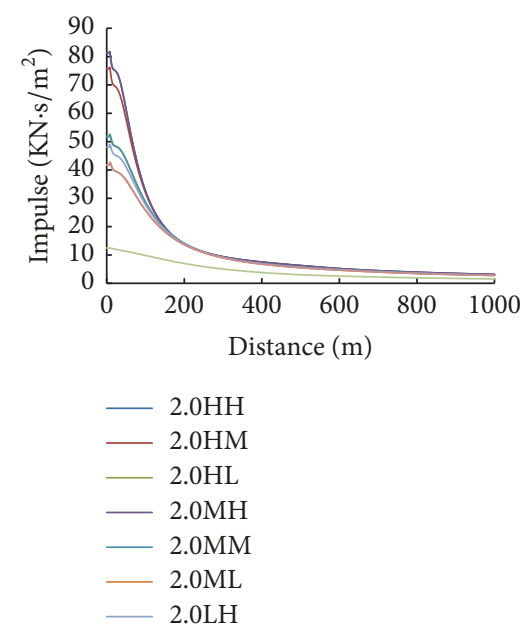

(h) 200 tons

FIGURE 5: Impulse versus distance for various flammable masses. 


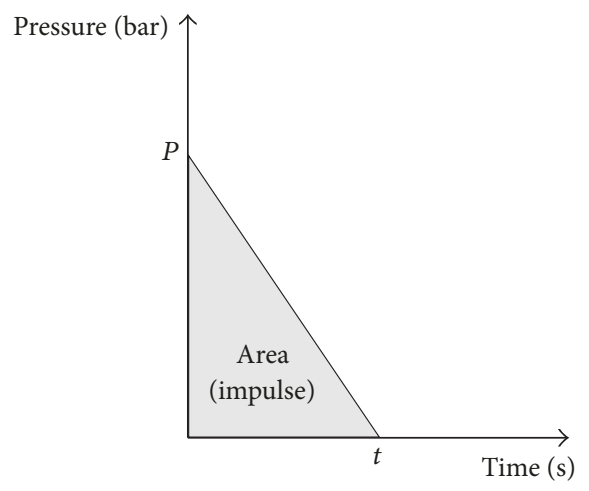

FIGURE 6: Representative blast pressure model.

TABLE 3: Weather and atmospheric conditions for Spring season (after Pintarič [27]).

\begin{tabular}{lc}
\hline Wind speed & $2 \mathrm{~m} / \mathrm{s}$ \\
Pasquill stability & D, neutral, little sun and high wind \\
Atmospheric temperature & 13 \\
Relative humidity & 0.7 \\
\hline
\end{tabular}

a direction perpendicular to the surface of the explosive and can be assumed to reach in the left front side of the structure (see Figure 2). In order to understand the blast behavior of the weir structure in terms of flame speed based on the BST model, nine (9) blast-load scenarios with various congestion and material reactivity conditions were considered in the linear elastic FE weir model with the nonlinear soil-structure interaction model based on the isotropic Coulomb friction material. For instance, Figure 7 shows stress-time history for the blast-wave expansion process with time domain using the distance and flammable masses selected as zero meters and 5 tons with high congestion and material reactivity conditions for more rigorous and conservative analysis. At the very beginning of the explosion, the maximum principal stress due to the strong shockwave was $4.0 \mathrm{MPa}$, and subsequently the high blast shockwave stress occurred on the area of the weir. And, then the maximum stress was significantly concentrated on the area of connection between the weir body and the stilling basin as time went by. It tells us that the initial crack may develop the left side of the weir, and the significant damage can occur at the right side of the weir hitting the stilling basin by the shockwave. Figure 8 shows the simulation results on the tensile and compressive stresses for the weir using various types of congestion levels and material relativities: HH, HM, HL, MH, MM, ML, LH LM, and LL. The maximum tensile and compressive stresses for were found $16.38 \mathrm{MPa}$ and $11.61 \mathrm{MPa}$ under the $\mathrm{HH}(\mathrm{MH})$ and LM (ML) conditions, respectively. As for the absolute maximum displacement (LL condition), it was $16.83 \mathrm{~mm}$ at the end of the stilling basin, as can be seen in the figure. Correspondingly, the nonlinear response of displacements for the stilling basin should be checked independently from the pressure-time-history data for a strong shockwave analysis since the maximum displacement was not obtained as the same congestion and material reactivity conditions as the maximum pressure.

Furthermore, in order to understand the blast behaviors of the system according to the uncertainties of flammable mass and stand-off distance, eight flammable masses $(5,10$, $20,40,60,80,100$, and 200 tons) and six stand-off distances $(100,200,400,600,800$, and $1000 \mathrm{~m})$ under the high congestion and material reactivity conditions were considered in this study. For the low-flammable-mass levels, the tensile stresses were greater than the compressive stresses, and then tensile and compressive stresses became closed as mass levels increased as shown in Figure 9(a). The relationships between tensile and compressive stresses as a function of $x$ (mass) were found to be $0.033 x+19.44$ and $0.025 x+$ 16.01, respectively. Figure 9(b) shows the maximum tensile and compressive stresses versus various types of stand-off distances. Both tensile and compressive stresses increased as the stand-off distances increased, and stresses were converged at the distance of $800 \mathrm{~m}$. The relationships between tensile and compressive stresses as a function of $x$ (distance) were found to be $-1 E-5 x^{2}+0.021 x+2$ and $-1 E-5 x^{2}+0.020 x+2$, respectively. It should be noted that the tensile stress, compressive stress, and displacement were gradually increased with increasing explosive mass and stand-off distance.

Figure 10 shows the results in terms of the shockwave $(5 t, \mathrm{HH})$-induced stress changes of the weir in the linear and nonlinear soil-structure interaction models. As seen in the time histories, the compressive stress at the connection area shows similar trends, whereas the tensile stress was significantly different between the two models. Specifically, the tensile stress of the linear soil-structure interaction model was much higher than that of the nonlinear model. It shows us some amount of energy was consumed in nonlinear behavior under the blast loading with nonlinear soil-structure interaction. Finally, sensitivity study for the effects of the friction for the weir system were conducted as shown in Figure 11. The friction coefficients were increased from 0.7 to 0.9 considering the uncertainty of concrete on concrete frictional condition. The results showed the friction effect on the weir structure subjected to high-intensity blast loading was not significant as shown the mean tensile and compressive stress values were $3.96 \mathrm{MPa}$ and $4.36 \mathrm{MPa}$ with mean plus one standard deviation indications, respectively.

\section{Conclusions}

This study evaluated the blast response and possible damage locations of weir structures under high-intensity loadings such as blast shock waves. In order to define the blast loads and account for the material reactivity and congestion levels, the PHAST program was used. Also, the parameters for the BST models represented the relevant weather data and atmospheric factors for a given area of explosion. Additionally, for structural simulation under blast shockwave, the ABAQUS platform was adapted to develop the linear elastic FE weir model with the nonlinear soil-structure interaction model based on the isotropic Coulomb friction material. Various parametric studies were conducted to investigate the different blast responses of the concrete flood-defense system under 


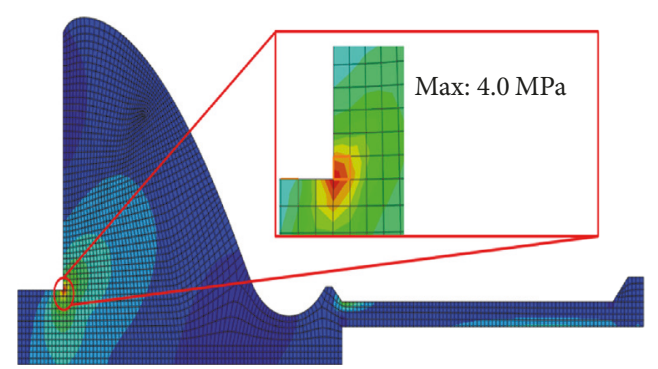

(a) Maximum principal stress at $0.006 \mathrm{~s}$

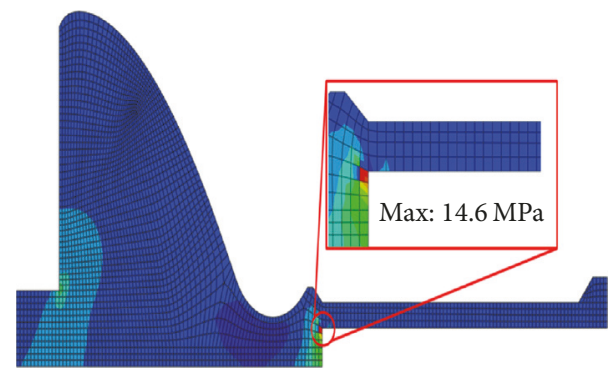

(c) Maximum principal stress at $0.176 \mathrm{~s}$

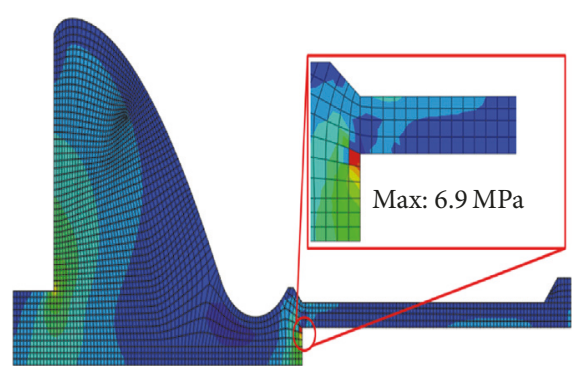

(b) Maximum principal stress at $0.012 \mathrm{~s}$

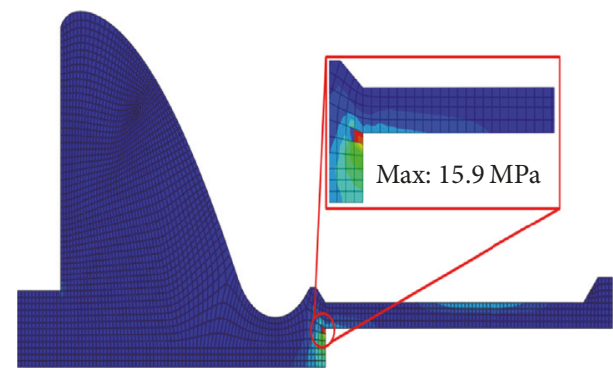

(d) Maximum principal stress at $0.235 \mathrm{~s}$

FIGURE 7: Blast-wave expansion process with time domain (5 tons, $\mathrm{HH}$ ).

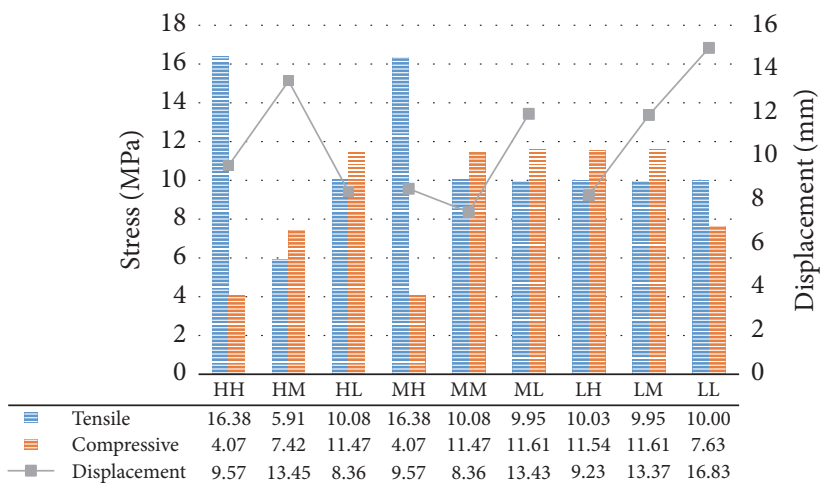

FIGURE 8: Results' comparison based on material reactivity and congestion levels.

different blast conditions, stand-off distance, and friction coefficients: nine blast-load scenarios with various congestion and material reactivity conditions $(\mathrm{HH}, \mathrm{HM}, \mathrm{HL}, \mathrm{MH}$, MM, ML, LH LM, and LL), eight flammable masses $(5,10,20$, $40,60,80,100$, and 200 tons), six stand-off distances (100, 200, $400,600,800$, and $1000 \mathrm{~m}$ ), and three friction coefficients $(0.7,0.8$, and 0.9$)$. The blast shockwave data and observations obtained in this study and presented herein can be used to support the development of comprehensive regulatory design guidelines for the design and development of concrete weir structures subject to various threat-scenario uncertainties. The primary research results in this study are listed as follows:

(i) The initial crack may develop the left side of the weir, and the significant damage can occur at the right side of the weir (between the concrete weir body and stilling basin) hitting the stilling basin by the shockwave. (ii) The blast damage to concrete weir structure was significantly influenced by congestion levels or material reactivity.

(iii) The tensile and compressive stress levels were sensitive to the flammable-mass levels, and both tensile and compressive stresses increased as the stand-off distances increased and were converged at the certain distance. The tensile stress, compressive stress, and displacement were gradually increased with increasing explosive mass and stand-off distance.

(iv) Some amount of energy was consumed in nonlinear behavior under the blast loading when nonlinear soilstructure interaction was considered in an analytical model.

(v) Sensitivity study was conducted for the effects of using the different value of friction coefficients for the weir 


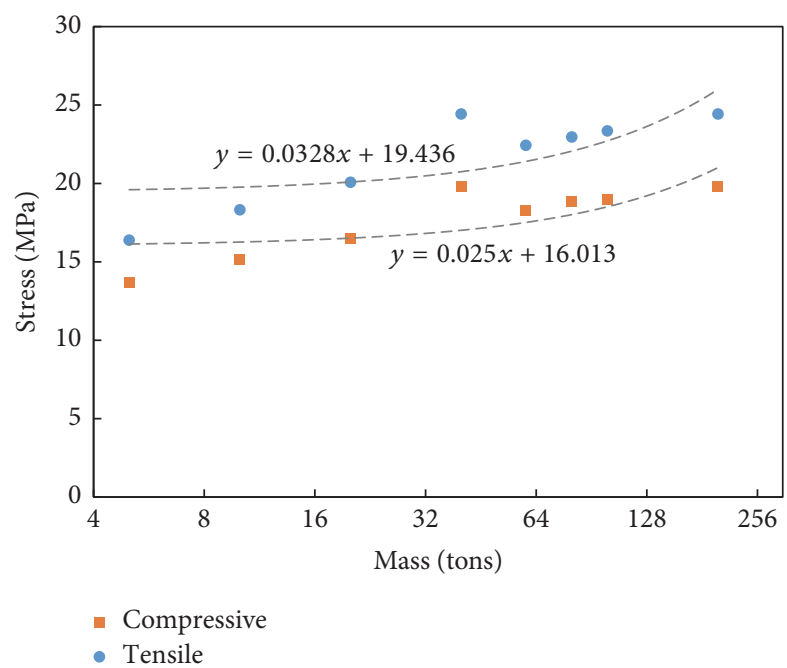

(a) Stresses based on explosive masses

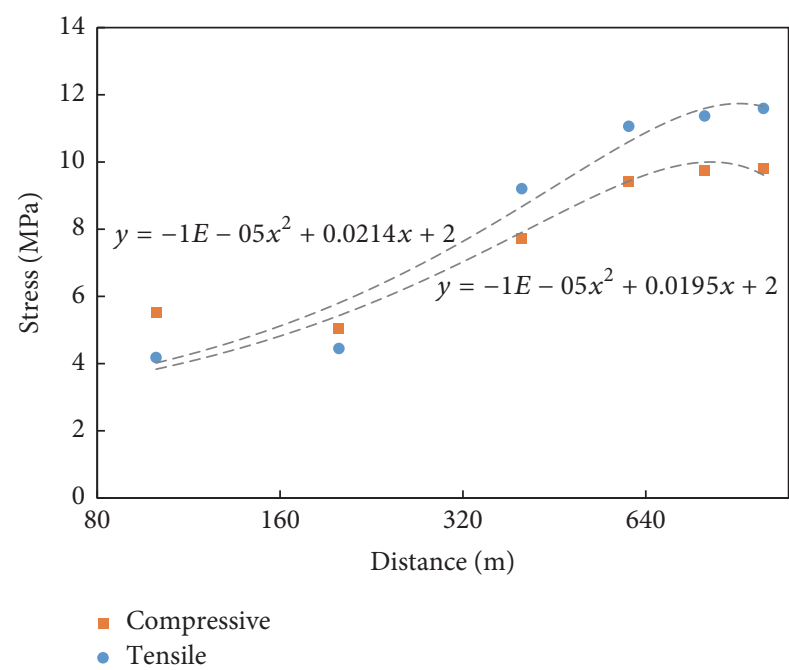

(b) Stresses based on stand-off distances

FIGURE 9: Stress changes of the weir in accordance with explosive masses and stand-off distances.

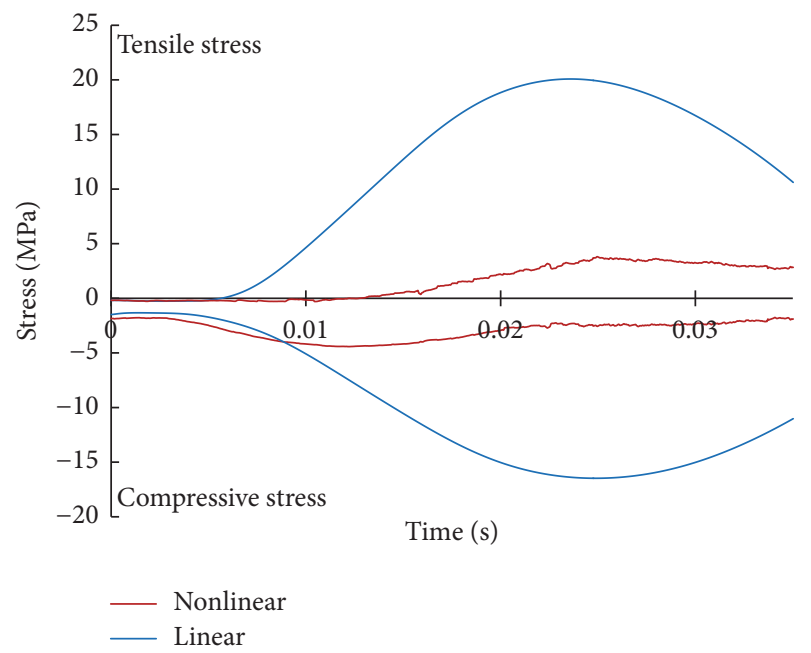

FIgURE 10: Blast vibration of the weir associated with linear and nonlinear soil-structure interaction models.

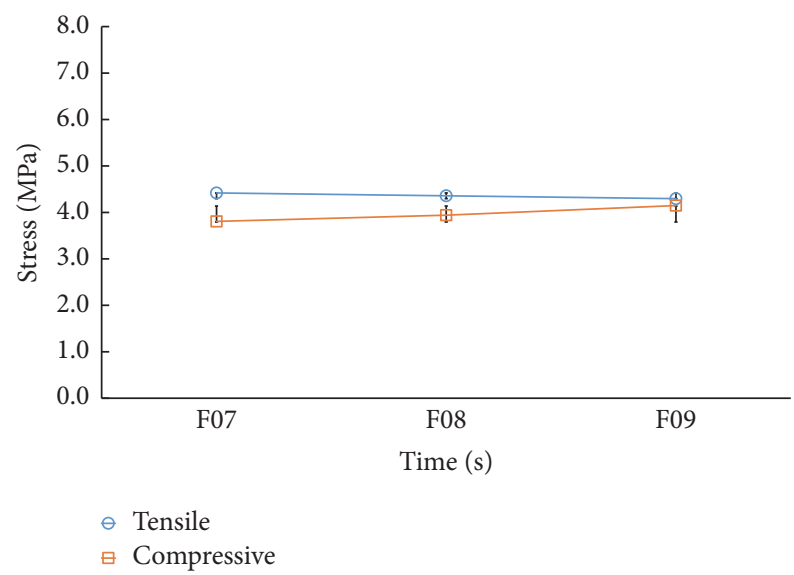

FIGURE 11: Sensitivity study with respect to friction coefficients. system. As a result, it was revealed that the effect of the friction on the weir system under the blast loading was not significant.

\section{Conflicts of Interest}

The authors declare that there are no conflicts of interest regarding the publication of this paper.

\section{Acknowledgments}

This work was supported by a grant from Kyung Hee University in 2016 (KHU-20161374).

\section{References}

[1] G. Razaqpur, W. Mekky, and S. Foo, "Fundamental concepts in blast resistance evaluation of structures," Canadian Journal of Civil Engineering, vol. 36, no. 8, pp. 1292-1304, 2009.

[2] E. Jacques, A. Lloyd, and M. Saatcioglu, "Predicting reinforced concrete response to blast loadsl," Canadian Journal of Civil Engineering, vol. 40, no. 5, pp. 427-444, 2013.

[3] C. Viau and G. Doudak, "Investigating the behaviour of typical and designed wall-to-floor connections in light-frame wood stud wall structures subjected to blast loading," Canadian Journal of Civil Engineering, vol. 43, no. 6, pp. 562-572, 2016.

[4] T. Nog, P. Mendis, A. Gupta, and J. Ramsay, "Blast loading and blast effects on structures-an overview," EJSE Special Issue: Loading on Structure, pp. 76-91, 2007.

[5] K. P. Dharmasena, H. N. G. Wadley, Z. Xue, and J. W. Hutchinson, "Mechanical response of metallic honeycomb sandwich panel structures to high-intensity dynamic loading," International Journal of Impact Engineering, vol. 35, no. 9, pp. 10631074, 2008.

[6] H. Y. Low and H. Hao, "Reliability analysis of reinforced concrete slabs under explosive loading," Structural Safety, vol. 23, no. 2 , pp. 157-178, 2001. 
[7] Z. Xiangdi, J. Chunming, W. Yanping, Y. Jiwu, and W. Zheng, "Simulation study on an explosion accident in China," Process Safety Progress, vol. 33, no. 1, pp. 56-63, 2014.

[8] S. Fujikura, M. Bruneau, and D. Lopez-Garcia, "Experimental investigation of multihazard resistant bridge piers having concrete-filled steel tube under blast loading," Journal of Bridge Engineering, vol. 13, no. 6, pp. 586-594, 2008.

[9] Federal Emergency Management Agency (FEMA), Reference Manual to Mitigate Potential Terrorist Attacks against Buildings, FEMA 426, Washington D.C, Wash, USA, 2003.

[10] Federal Highway Administration (FHWA), Recommendations for Bridges and Tunnel Security, the Blue Ribbon Panel on Bridge and Tunnel Security, Washington D.C, Wash, USA, 2003.

[11] G. Li, P. Summers, K. Clutter, and D. Bonaventure, "Blast and impact resistant design of overhead protection structures," in Proceedings of the Structures Congress 2012, pp. 167-176, USA, March 2012.

[12] "PHAST Professional Version 7.1; Risk Management software; DNV Technica, London".

[13] H. W. M. Witlox, M. Harper, and A. Oke, "Phast validation of discharge and atmospheric dispersion for carbon dioxide releases," in Proceedings of the 15th Annual Symposium, Mary Kay O'Connor Process Safety Centre, Texas A\&M University College Station, Texas, TX, USA, 2012.

[14] H. W. M. Witlox and A. Oke, Verification and Validation of Consequence Models for Accidental Releases of Hazardous Chemicals to the Atmosphere, vol. 154 of Institution of Chemical Engineers Symposium Series, Institution of Chemical Engineers; 1999, 2008.

[15] DNV software, "Obstructed Region Explosion Model Validation," Phast 7.1 technical documentation, 2010.

[16] A. J. Pierorazio, J. K. Thomas, Q. A. Baker, and D. E. Ketchum, "An update to the Baker-Strehlow-Tang vapor cloud explosion prediction methodology flame speed table," Process Safety Progress, vol. 24, no. 1, pp. 59-65, 2005.

[17] Y. Xu, D. Worthington, and A. Oke, Correcting the Predictions by Baker-Strehlow-Tang (BST) Model for the Ground Effect, Symposium Series No. 155, IChemE, 2009.

[18] ABAQUS, "Ver 6.13, Dassault Systemes".

[19] B. S. Ju and W. Jung, "Evaluation of seismic fragility of weir structures in South Korea," Mathematical Problems in Engineering, vol. 2015, Article ID 391569, 10 pages, 2015.

[20] B. S. Ju and W. Y. Jung, "Evaluation of seismic performance of weir structures in Korea," International Journal of Engineering and Technology (IJET), vol. 7, no. 2, pp. 808-816, 2015.

[21] H. M. Westergaard, "Water pressure on dams during earthquakes," Transactions of the American Society of Civil Engineers, vol. 98, pp. 418-433, 1933.

[22] O. R. Hansen, R. Martini, J. Choi, and Y. Ryu, "Impact of DDT on FPSO explosion risk assessment," Process Safety Progress, vol. 34, no. 1, pp. 44-57, 2015.

[23] G. Atkinson, "Blast damage to storage tanks and steel clad buildings," Process Safety and Environmental Protection, vol. 89, no. 6, pp. 382-390, 2011.

[24] CSB, "Final Investigation Report Refinery Explosion and Fire, US Chemical Safety and Hazard Investigation Board," 2007, http://www.csb.gov/assets/1/19/csbfinalreportbp.pdf.

[25] Q. A. Baker, C. M. Doolittle, G. A. Fitzgerald, and M. J. Tang, Recent Developments in the Baker-Strehlow VCE Analysis Methodology, Loss Prevention Symposium, AICHE, 31st edition, 1997, paper 42f.
[26] M. J. Tang and Q. A. Baker, "A new set of blast curves from vapor cloud explosion," Process Safety Progress, vol. 18, no. 4, pp. 235240, 1999.

[27] Z. N. Pintarič, "Assessment of the consequences of accident scenarios involving dangerous substances," Process Safety and Environmental Protection, vol. 85, no. 1 B, pp. 23-38, 2007. 


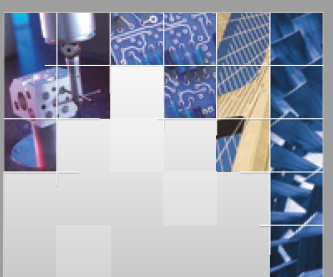

\section{Enfincering}
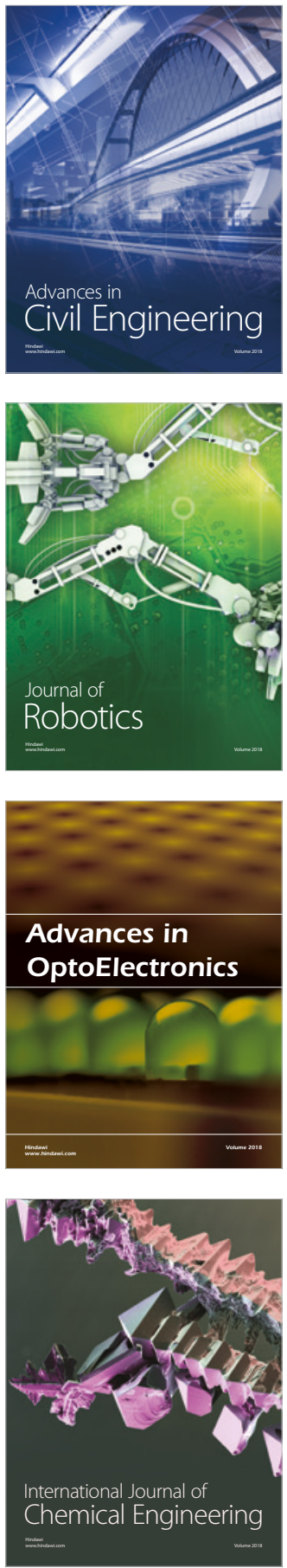

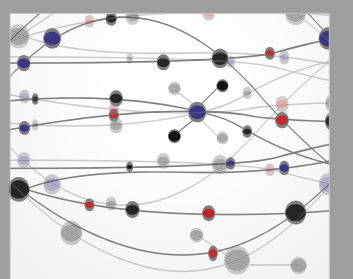

\section{Rotating \\ Machinery}

The Scientific World Journal

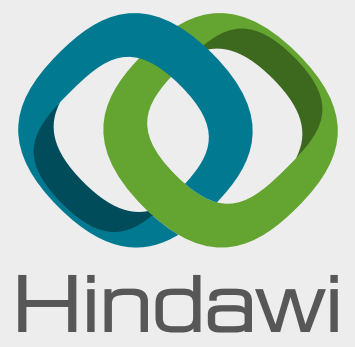

Submit your manuscripts at

www.hindawi.com
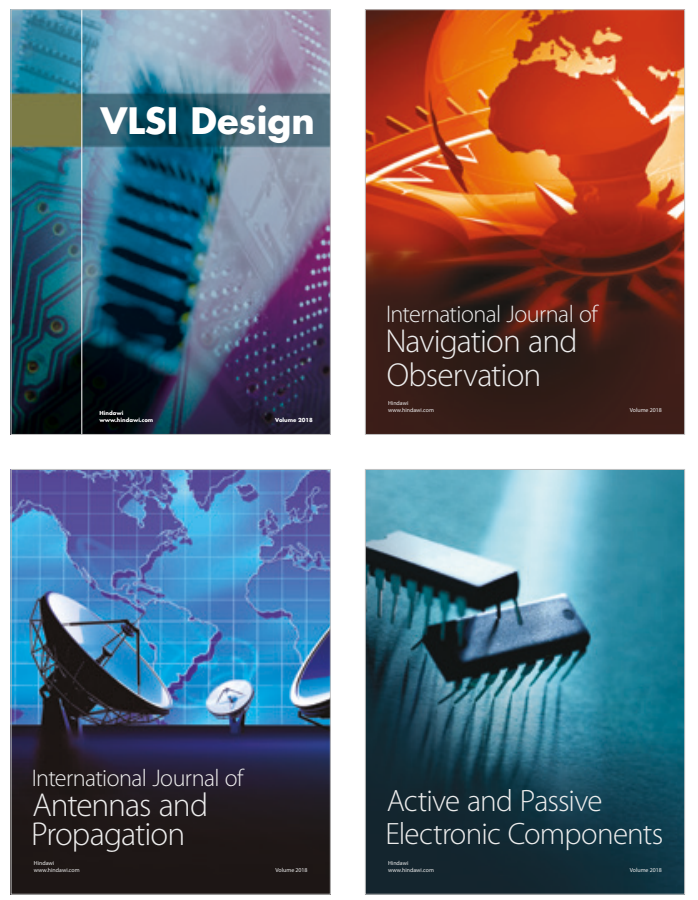
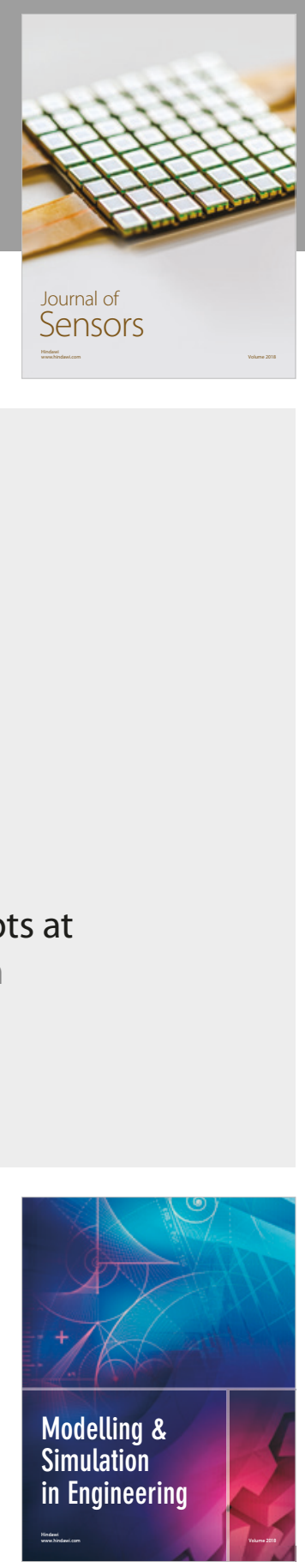

\section{Advances \\ Multimedia}
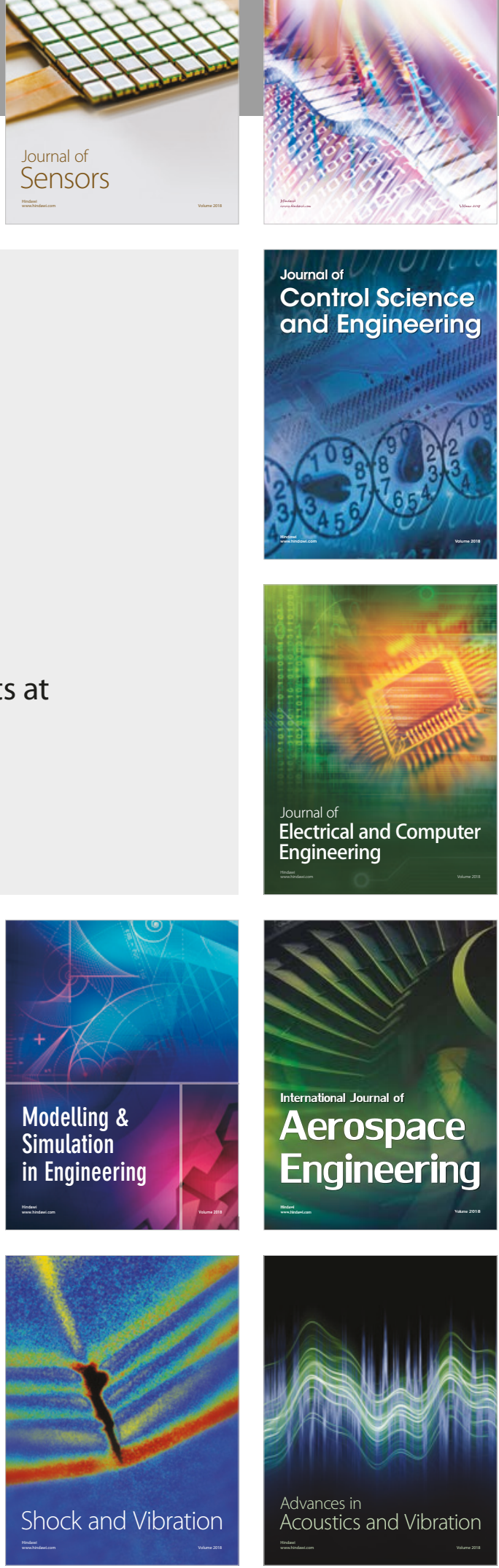